Check for updates

Cite this: RSC Adv., 2018, 8, 35157

Received 22nd May 2018

Accepted 3rd October 2018

DOI: $10.1039 / \mathrm{c} 8 \mathrm{ra0} 4347 \mathrm{~g}$

rsc.li/rsc-advances

\section{Alleviate the $J-V$ hysteresis of carbon-based perovskite solar cells via introducing additional methylammonium chloride into $\mathrm{MAPbl}_{3}$ precursor $\uparrow$}

\author{
Huirong Jiang, ${ }^{a}$ Xingyu Liu, ${ }^{a}$ Nianyao Chai, ${ }^{a}$ Fuzhi Huang, (D) ${ }^{a}$ Yong Peng, ${ }^{a}$ \\ Jie Zhong, (D) a Qi Zhang, (D)*ac Zhiliang Ku (D)*ab and Yi-bing Cheng ${ }^{a}$
}

The hysteretic phenomenon commonly exists in the $J-V$ curves of perovskite solar cells with different structures, especially for carbon-based mesoscopic perovskite solar cells without hole-conductor (carbon-based PSCs). By adding moderate amounts of methylammonium chloride (MACl) into $\mathrm{MAPbl}_{3}$ perovskite precursor, we found the $J-V$ hysteresis of carbon-based PSCs could be significantly alleviated and the crystallinity of $\mathrm{MAPbl}_{3}$ perovskite could also be influenced. With the increasing amount of $\mathrm{MACl}$, $\mathrm{MAPb}_{3}$ perovskite showed better and better crystallinity until the $\mathrm{MACl}$ came to $0.45 \mathrm{M}$. The champion device with $0.45 \mathrm{M}$ of additional $\mathrm{MACl}$ exhibited a preferable PCE of $14.27 \%$ for reverse-scan (RS) and $14.50 \%$ for forward-scan (FS), significantly higher than that of the pristine device $(8.74 \%$ for RS and $4.80 \%$ for FS). What's more, the $J-V$ hysteretic index of the device gradually decreased along with the increasing amount of $\mathrm{MACl}$, and kept at low value even when the crystallinity of $\mathrm{MAPbl}_{3}$ perovskite became poor. Through XRD and $\mathrm{PL}$ analysis, we demonstrated that the recombination rate of the accumulated charges at the perovskite/ $/ \mathrm{TiO}_{2}$ interface is the main reason for photocurrent hysteresis in carbon-based PSCs. High quality of perovskite crystals is an important contributing factor for highperformance PSCs with low hysteresis, but there is no necessary correlation between low hysteresis and good crystallinity. This research presents an effective way to fabricate carbon-based PSCs with lowhysteresis, and at the same time, provides evidence for investigating the origin of $J-V$ hysteresis of PSCs.

\section{Introduction}

In the past few years, the organic-inorganic hybrid lead halide perovskite materials (e.g. methylammonium lead iodide, $\mathrm{MAPbI}_{3}$ ) have caught world-wide attention for their excellent photo- and electrical-properties such as high absorption coefficient, long charge diffusion length and high carrier mobility. ${ }^{\mathbf{1 - 5}}$ Solar cells based on these perovskite light absorbers (perovskite solar cells, PSCs) have shown tremendous application potential due to their easy fabrication procedures and high power conversion efficiency (PCE). Up to now, the world champion PCE of PSCs has reached $22.7 \%$, far exceeding that of the other emerging photovoltaic technologies such as dye-

${ }^{a}$ State Key Laboratory of Advanced Technologies for Materials Synthesis and Processing, International School of Materials Science and Engineering, Wuhan University of Technology, 122 Luoshi Road, Wuhan, Hubei, P. R. China. E-mail: zhiliang.ku@whut.edu.cn

${ }^{b}$ Hubei Key Laboratory of Low Dimensional Optoelectronic Material and Devices, Hubei University of Arts and Science, 296 Longzhong Road, Xiangyang, Hubei Province, P. R. China

${ }^{c}$ School of Aerospace, Transport and Manufacturing, Cranfield University, Cranfield, Bedfordshire, MK430AL, UK. E-mail: q.zhang@cranfield.ac.uk

$\dagger$ Electronic supplementary information (ESI) available: IPCE spectra of the champion devices. See DOI: 10.1039/c8ra04347g sensitized solar cells (DSSCs) and organic photovoltaics (OPV). ${ }^{6}$ For most kinds of the PSCs, they still need Au or Ag as their counter electrodes, which are commonly deposited by thermal evaporation. However, the usage of noble metals, together with high vacuum facilities in the fabrication procedures, is unfavourable for achieving a low cost of PSCs. In this regard, carbon-based PSCs, ${ }^{7}$ which employ carbon as counter electrode, has significant research value for low-cost PSCs. What's more, the carbon-based PSCs can be fabricated via fullyprinting procedures, which is propitious for scaling-up the devices. Since firstly be reported in 2013, carbon-based PSCs have been studied by many research groups in aspect of materials, interface engineering, solvent engineering and long-term stability. ${ }^{8-15}$ However, like other types of PSCs, carbon-based PSCs suffer from the current density-voltage $(J-V)$ hysteresis as well. The $J-V$ hysteresis influences the PCE of a solar cell device and occurs as a result of the large discrepancy between the forward and reverse scans. Though researchers haven't come to an agreement on the origin of this anomalous hysteretic behaviour in PSCs, most of them considered that ion migration, ${ }^{16,17}$ ferroelectricity, ${ }^{18,19}$ a photo-induced capacitive effect $^{20,21}$ and charge trapping/detrapping ${ }^{22,23}$ could be the reasons. For optimum PCE, several strategies ${ }^{\mathbf{8 , 1 2 , 1 4 , 1 5}}$ have been applied on the carbon-based PSCs. These strategies can also 
alleviate the hysteretic behaviour of the device, and most of the researchers believe that poor crystallinity of perovskite should be the culprit of hysteresis in carbon-based PSCs.

In this work, we mainly focused on the $J-V$ hysteretic behaviour of the carbon-based PSCs and investigated the reason for $J-V$ hysteresis. By adding extra methylammonium chloride (MACl) into $\mathrm{MAPbI}_{3}$ perovskite precursor, we found that the $J-V$ hysteresis of the device disappeared gradually as the increasing amount of MACl. For dug reason, the X-ray diffraction (XRD) and steadystate photoluminescence (PL) measurements were performed on the devices. The XRD results showed that the crystallinity of $\mathrm{MAPbI}_{3}$ perovskite can be improved by certain amount of $\mathrm{MACl}$, and better crystallinity also brings higher PCE to the device. The champion device exhibited a reverse-scan (RS) PCE of 14.27\% and forward-scan (RS) PCE of $14.50 \%$, which was significantly higher than that of the pristine device $(8.74 \%$ for RS and $4.80 \%$ for FS). Furthermore, with continuous increasing the amount of $\mathrm{MACl}$, the crystallinity of $\mathrm{MAPbI}_{3}$ perovskite got worse and worse. However, the $J-V$ hysteresis of the devices still kept at low level. PL results revealed that the recombination rate of the accumulated charges at the perovskite/ $\mathrm{TiO}_{2}$ interface is the main reason for photocurrent hysteresis. Hence, to fabricate hysteresis-free carbon-based PSCs with high performance, optimizing the perovskite/ $\mathrm{TiO}_{2}$ interface should be the priority concern.

\section{Experimental}

\section{Materials and precursor preparation}

MAI, $\mathrm{PbI}_{2}, \mathrm{MACl}$ and FACl were purchased from Xi'an p-OLED Corp. $N, N$-Dimethyl formamide (DMF) were purchased from Sigma-Aldrich. $\mathrm{TiO}_{2}$ paste was purchased from NJUKII. $\mathrm{ZrO}_{2}$ and carbon paste were prepared as former reports. ${ }^{7}$

The pristine perovskite precursor was prepared by dissolving the mixture composed of $461.01 \mathrm{mg} \mathrm{PbI}_{2}$ and $158.97 \mathrm{mg}$ MAI in $1 \mathrm{~mL}$ DMF to achieve $1 \mathrm{M} \mathrm{MAPbI}_{3}$ precursor solution.

The MACl-incorporated perovskite precursor was prepared via adding $x$ mmol MACl $(x=0.15,0.3,0.45,0.6,0.75,0.9,1.05)$ into $1 \mathrm{~mL}$ pristine perovskite precursor to achieve $\mathrm{MAPbI}_{3}$ with $x$ M MACl precursor solution.

\section{Device fabrication}

FTO glass was etched by a laser machine (Universal Laser Systems, VLS2.30), and followed by ultrasonic cleaning with detergent solution, pure water and ethyl alcohol for $20 \mathrm{~min}$, respectively. A compact $\mathrm{TiO}_{2}$ layer was deposited on FTO glass by spray pyrolysis deposition at $450{ }^{\circ} \mathrm{C}$ with a 2-propanol solution involving titanium diisopropoxide bis(acetylacetonate) solution. A $1 \mu \mathrm{m}$ mesoscopic $\mathrm{TiO}_{2}$ layer (NJU-30NR-D) was screen-printed on top of the compact layer and then sintered at $500{ }^{\circ} \mathrm{C}$ for $30 \mathrm{~min}$. After that, a $1.6 \mu \mathrm{m} \mathrm{ZrO}_{2}$ and a $11 \mu \mathrm{m}$ carbon layer were subsequently screen-printed above the $\mathrm{TiO}_{2}$ mesoscopic layer. The $\mathrm{ZrO}_{2}$ layer and carbon layer ware sintered at $500{ }^{\circ} \mathrm{C}$ and $400{ }^{\circ} \mathrm{C}$ for $30 \mathrm{~min}$, respectively. After cooling down to room temperature, the triple layer was infiltrated with $5 \mu \mathrm{L}$ perovskite solution via drop casting on top of the carbon counter electrode and then annealed at $100{ }^{\circ} \mathrm{C}$ for $20 \mathrm{~min}$. All the above procedures were accomplished in air atmosphere.

\section{Characterization}

The XRD spectra were recorded on a Bruker D8 Advance X-ray diffractometer with $\mathrm{Cu} \mathrm{K} \alpha$ radiation $(\lambda=1.5418 \AA)$. The UVvisible spectra of perovskite films deposited on glass were measured by PerkinElmer lambda 750S spectrophotometer. The cross-sectional structure and elemental distribution of the perovskite solar cells were investigated with field-emission scanning electron microscopy (FE-SEM, Zeiss Ultra Plus) equipped with an EDS detector. Steady-state photoluminescence spectra were measured by a PL microscopic spectrometer (Flex One, Zolix, China) with an excitation wavelength of $532 \mathrm{~nm}$. Photocurrent density-voltage $(J-V)$ characteristics and steady-state output of photocurrent density were tested by a solar simulator (Oriel 94023A, $300 \mathrm{~W}$ ) and a Keithley 2400 source meter under AM $1.5 \mathrm{G}\left(100 \mathrm{~mW} \mathrm{~cm}^{-2}\right)$. The light intensity was calibrated by a standard Si solar cell (Oriel, VLSI standards). All the devices were tested under AM 1.5 G sun light $\left(100 \mathrm{~mW} \mathrm{~cm}^{-2}\right)$ using a black mask with an aperture area of $0.1475 \mathrm{~cm}^{2}$ at a scan rate of $0.1 \mathrm{~V} \mathrm{~s}^{-1}$. EQE measurements were performed by an internal establishment system with monochromatic light and white bias light ( $\sim 0.1$ sun). The photodiode used for the calibration of EQE measurements has been calibrated by Newport.

\section{Results and discussion}

Carbon-based PSCs were fabricated by the procedures reported previously. ${ }^{7}$ Briefly, via screen printing technique, mesoporous $\mathrm{TiO}_{2}, \mathrm{ZrO}_{2}$ and carbon films were successively deposited on the FTO substrates, which have been coated with compact $\mathrm{TiO}_{2}$ beforehand. Afterwards, $\mathrm{MAPbI}_{3}$ perovskite light absorber was loaded by filtrate the precursor solution into the mesoporous carbon $/ \mathrm{ZrO}_{2} / \mathrm{TiO}_{2}$ layers (shown in Fig. 1a). The microstructure of the as-prepared device was observed by SEM measurement from the cross-section, and we can find each layer has welldefined boundaries and uniform thickness (see Fig. 1b). To investigate how the $\mathrm{MACl}$ additive influences the performance of the device, we added different amount of MACl $(0.15,0.3$, $0.45,0.6,0.75,0.9,1.05 \mathrm{M})$ into the standard $1 \mathrm{M} \mathrm{MAPbI}_{3}$ precursor.

Photocurrent density-voltage $(J-V)$ characteristics (measured under AM $1.5 \mathrm{G}$ irradiation at $100 \mathrm{~mW} \mathrm{~cm}^{-2}$ with a scan rate of $10 \mathrm{mV} \mathrm{s}^{-1}$ ) of the devices assembled by using these precursors are shown in Fig. 2 and the detail parameters are summarized in Table S1. $\dagger$ Interestingly, with the adding of $\mathrm{MACl}$ from 0 to

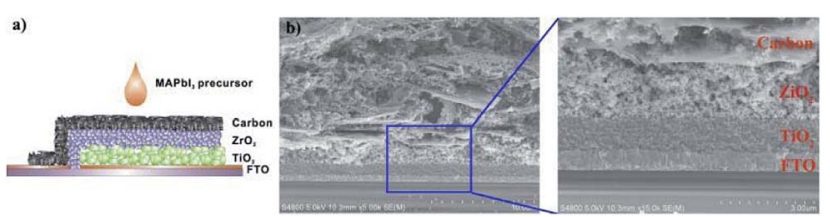

Fig. 1 (a) The schematic structure of the carbon-based PSCs; (b) SEM images from the cross section of the carbon-based PSCs. 

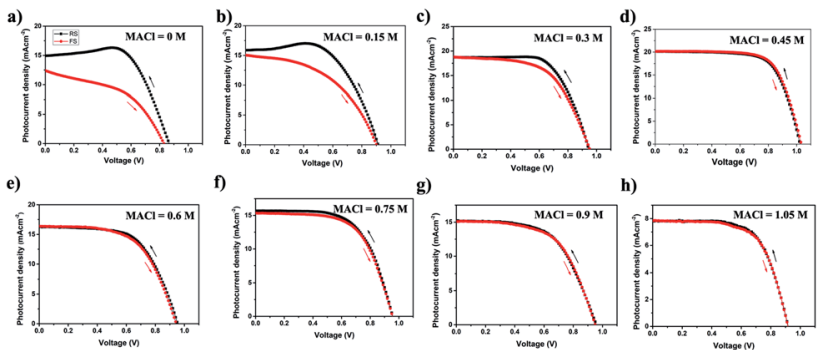

Fig. $2 \mathrm{~J}-V$ curves of $\mathrm{MAPb}_{3}$-based PSCs with different amount of $\mathrm{MACl}$.

$0.3 \mathrm{M}$, the $J-V$ hysteretic behaviour alleviated gradually (see Fig. 2a-c). When the amount of MACl came to $0.45 \mathrm{M}$, no obvious hysteresis can be found from the $J-V$ curve (see Fig. 2d) and the PCE of the device reached maximum value.

Fig. 3 presents the $J-V$ parameters of the corresponding champion carbon-based PSCs. The carbon-based PSCs with pristine $\mathrm{MAPbI}_{3}$ perovskite showed poor PCE and obvious $J-V$ hysteresis ( $8.74 \%$ for RS and $4.80 \%$ for FS). However, by adding $0.45 \mathrm{M} \mathrm{MACl}$ into $\mathrm{MAPbI}_{3}$ perovskite precursor, the champion device achieved a PCE of $14.27 \%$ with low $J-V$ hysteresis (IPCE spectra and long-term stability are shown in Fig. S1 and S2†). To ensure the improvement of $\mathrm{MACl}$ on the performance of PSCs device is repeatable, two batches of devices (each consist of 15 devices) were fabricated by $\mathrm{MAPbI}_{3}$ perovskite and $\mathrm{MAPbI}_{3} /$ $0.45 \mathrm{MACl}$, respectively. The devices with pure $\mathrm{MAPbI}_{3}$ possessed an average PCE of 7.09\% and the ones with $\mathrm{MAPbI}_{3} /$ $0.45 \mathrm{MACl}$ exhibited an average PCE of $13.36 \%$ (see Table S2 and $\mathrm{S} 3 \dagger)$.

As the continuous increasing of $\mathrm{MACl}$, the corresponding devices showed a tendency of lower PCE (see Fig. 2e-h). Note that, though with poorer PCEs, the $J-V$ hysteresis of devices still kept at low level. To make clear how MACl influence the $J-V$ hysteresis of the device, XRD measurement was utilized to investigate the crystallinity of $\mathrm{MAPbI}_{3}$ perovskite prepared by different precursors.

As shown in Fig. 4, all of the perovskite samples exhibit similar diffraction peaks at $2 \theta$ values of $13.91^{\circ}, 28.20^{\circ}$ and $31.69^{\circ}$, corresponding to the $(110),(220)$ and $(310)$ crystal planes of tetragonal $\mathrm{MAPbI}_{3}$ perovskite. With the adding of additional $\mathrm{MACl}$ from $0.15 \mathrm{M}$ to $0.45 \mathrm{M}$, the peak at $2 \theta$ value of $13.91^{\circ}$ gradually increased its intensity, indicating better and better

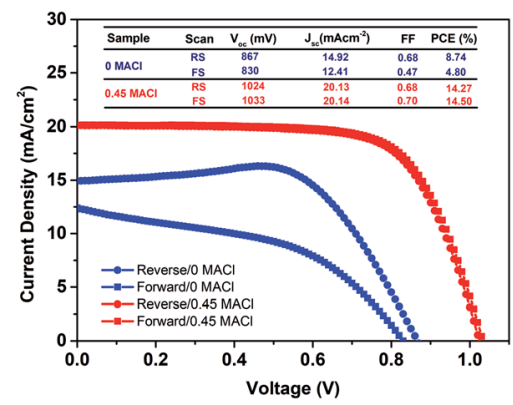

Fig. $3 \mathrm{~J}-\mathrm{V}$ curves of champion MAPbl3-based PSCs with 0 and $0.45 \mathrm{M} \mathrm{MACl}$.

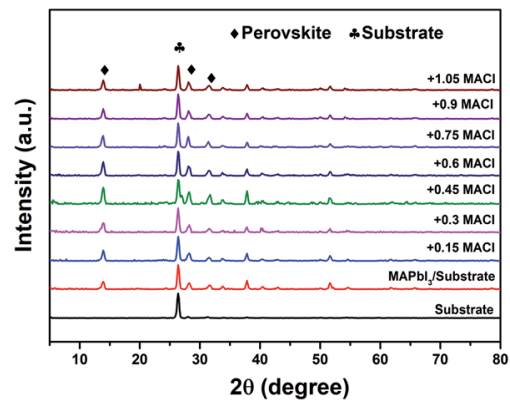

Fig. 4 XRD patterns of $\mathrm{MAPbl}_{3}$ with different amount of $\mathrm{MACl}$ on $\mathrm{FTO}$ substrates.

crystallinity of $\mathrm{MAPbI}_{3}$ perovskite. Obviously, the good crystallinity of perovskite in the mesoporous films is the prerequisite of high PCE. When we continue increased the amount of MACl (from $0.6 \mathrm{M}$ to $1.05 \mathrm{M}$ ), the crystallinity of $\mathrm{MAPbI}_{3}$ became worse again. Moreover, no obvious $\mathrm{MAPbCl}_{3}$ peaks can be found in these patterns, indicating most of the chloride was released in the annealing procedure or the chloride only exist in the boundaries of $\mathrm{MAPbI}_{3}$ crystals. To confirm this, energy dispersive X-ray spectroscopy (EDS) measurement was performed on the device with champion PCE.

From the cross-section of the sample (Fig. 5a), we can clearly find the elements of carbon (Fig. 5b), zirconium (Zr, Fig. 5c) and titanium (Ti, Fig. 5d) distribute homogeneously in the top, middle and bottom area, respectively. Oxygen (Fig. 5b) is mainly exists in the $\mathrm{ZrO}_{2}, \mathrm{TiO}_{2}$ and FTO substrate. For perovskite, elements of lead $(\mathrm{Pb})$ together with iodine $(\mathrm{I})$ and chlorine $(\mathrm{Cl})$ have a uniform distribution in carbon $/ \mathrm{ZrO}_{2} / \mathrm{TiO}_{2}$ layers. However, the atom ratio of $\mathrm{Pb}, \mathrm{I}$ and $\mathrm{Cl}$ is around $1: 2.56: 0.12$, indicating only a few $\mathrm{Cl}$ exist in $\mathrm{MAPbI}_{3}$ perovskite. Thus, we deduced that most of the $\mathrm{Cl}$ elements were removed in the form of MACl gas ${ }^{24}$ by annealing. Meanwhile, the crystallinity of $\mathrm{MAPbI}_{3}$ perovskite changed as the release of MACl. To further study the different $J-V$ hysteresis behaviour of MAClincorporated $\mathrm{MAPbI}_{3}$ perovskite, steady-state photoluminescence (PL) spectra measurements were carried out on the glass $/ \mathrm{ZrO}_{2} /$ perovskite and $\mathrm{FTO} / \mathrm{TiO}_{2} /$ perovskite samples.

As illustrated by Fig. 6, the steady-state PL spectra of $\mathrm{MAPbI}_{3}$ perovskite films with different amount of MACl displayed a PL emission peak at $\sim 780 \mathrm{~nm}$. The $\mathrm{MAPbI}_{3}$ with $0.45 \mathrm{M}$ MACl film on the glass $/ \mathrm{ZrO}_{2}$ substrate showed the strongest PL intensity, indicating fewer traps and defects within the perovskite crystal (Fig. 6a). These results correspond well with the XRD results. In addition, we also performed the steady-state PL test on the

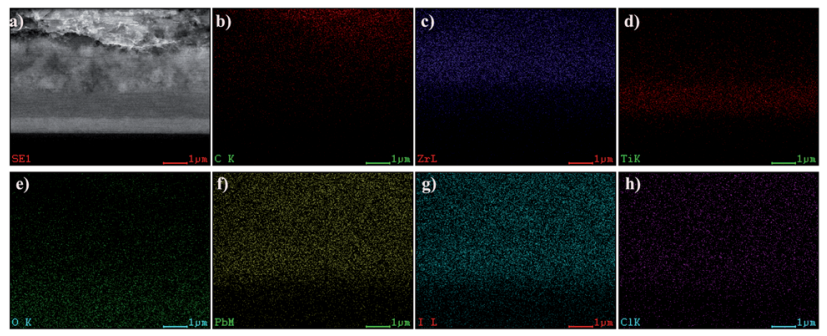

Fig. 5 EDS mapping of the carbon-based PSCs form cross-section. 

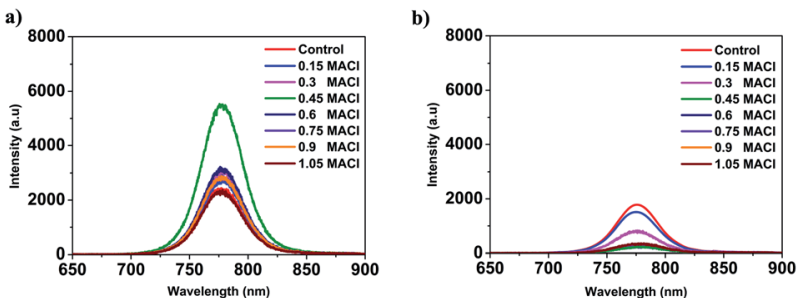

Fig. 6 The steady-state photoluminescence spectra of $\mathrm{MAPb}_{3}$ with different amount $\mathrm{MACl}$ on (a) glass $/ \mathrm{ZrO}_{2}$, (b) $\mathrm{FTO} / \mathrm{TiO}_{2}$.

perovskite samples with $\mathrm{FTO} / \mathrm{TiO}_{2}$ substrate (Fig. $6 \mathrm{~b}$ ). All of the $\mathrm{MAPbI}_{3}$ perovskite film with $\mathrm{MACl}$ exceeding $0.3 \mathrm{M}$ possessed a dramatically higher degree of PL quenching in comparison with other samples with lower amount of MACl. Generally, this PL quenching is attributed to originate from efficient electron extraction across the interface. ${ }^{25}$ Hence, we can conclude that the charge carrier extraction at the perovskite/ $\mathrm{TiO}_{2}$ interface could be enhanced by introducing sufficient $\mathrm{MACl}$ into $\mathrm{MAPbI}_{3}$. And $J-V$ hysteresis in carbon-based PSCs is mainly depends on the charge carrier extraction speed rather than the crystallinity of perovskite.

\section{Conclusions}

In summary, we demonstrated a facile method to alleviate the $J-$ $V$ hysteresis of carbon-based PSCs by introducing moderate $\mathrm{MACl}$ into $\mathrm{MAPbI}_{3}$ precursor. We adjusted the amount of $\mathrm{MACl}$ (from $0 \mathrm{M}$ to $1.05 \mathrm{M}$ ) in $\mathrm{MAPbI}_{3}$ precursor and found that the crystallinity of $\mathrm{MAPbI}_{3}$ appeared regularly change along with the amount of MACl. The champion device with the best crystallinity $\left(\mathrm{MAPbI}_{3}\right.$ with additional $0.45 \mathrm{M} \mathrm{MACl}$ in the precursor) achieved a PCE of $14.27 \%$ with low $J-V$ hysteresis, which is much higher than that of the pristine one (8.74\%). EDS results showed there was only a small amount of chloride residues in the $\mathrm{MAPbI}_{3}$ perovskite after annealing. We deduced that most of the chloride were removed in the form of MACl gas in the annealing procedure and thus, changed the crystallinity of $\mathrm{MAPbI}_{3}$ perovskite. Moreover, the steady-state PL measurements manifested that, with sufficient MACl in the $\mathrm{MAPbI}_{3}$ precursor, the charge carrier extraction at the perovskite/ $/ \mathrm{TiO}_{2}$ interface could be enhanced. Note that, for the carbon-based PSCs, there is no significant relationship between the interfacial charge extraction and the crystal quality of perovskite. These results would provide evidence for further investigating the origin of $J-V$ hysteresis in PSCs and solutions for fabricating high-performance carbon-based PSCs.

\section{Conflicts of interest}

There are no conflict to declare.

\section{Acknowledgements}

The authors acknowledge the financial support by National Natural Science Foundation of China (NSFC 51702243, 51402115, 51672202), Hubei Provincial Natural Science
Foundation of China (2016CFB464), the Technological Innovation Key Project of Hubei Province (2018AAA048), the Fundamental Research Funds for the Central Universities (WUT: 2016IVA093, 2016IVA089, 2016IVA085, 2016III030, 2017III022) and Hubei Key Laboratory of Low Dimensional Optoelectronic Material and Devices (HLOM151001).

\section{Notes and references}

1 A. Kojima, K. Teshima, Y. Shirai and T. Miyasaka, J. Am. Chem. Soc., 2009, 131, 6050-6051.

2 M. M. Lee, J. Teuscher, T. Miyasaka, T. N. Murakami and H. J. Snaith, Science, 2012, 338, 643-647.

3 H. S. Kim, C. R. Lee, J. H. Im, K. B. Lee, T. Moehl, A. Marchioro, S. J. Moon, R. Humphry-Baker, J. H. Yum, J. E. Moser, M. Gratzel and N. G. Park, Sci. Rep., 2012, 2, 591. 4 C. Wehrenfennig, G. E. Eperon, M. B. Johnston, H. J. Snaith and L. M. Herz, Adv. Mater., 2014, 26, 1584-1589.

5 G. C. Xing, N. Mathews, S. Y. Sun, S. S. Lim, Y. M. Lam, M. Gratzel, S. Mhaisalkar and T. C. Sum, Science, 2013, 342, 344-347.

6 N. R. E. L. N. Best Research Cell_Efficiencies, https:// www.nrel.gov/pv/assets/images/efficiency-chart.png, October, 2017.

7 Z. L. Ku, Y. G. Rong, M. Xu, T. F. Liu and H. W. Han, Sci. Rep., 2013, 3, 3132.

8 A. Mei, X. Li, L. Liu, Z. Ku, T. Liu, Y. Rong, M. Xu, M. Hu, J. Chen, Y. Yang, M. Gratzel and H. Han, Science, 2014, 345, 295-298.

9 G. Grancini, C. Roldán-Carmona, I. Zimmermann, E. Mosconi, X. Lee, D. Martineau, S. Narbey, F. Oswald, F. De Angelis, M. Graetzel and M. K. Nazeeruddin, Nat. Commun., 2017, 8, 15684.

10 M. Wang, W.-L. Yim, P. Liao and Y. Shen, ChemistrySelect, 2017, 2, 4469-4477.

11 A. K. Baranwal, S. Kanaya, T. A. Peiris, G. Mizuta, T. Nishina, H. Kanda, T. Miyasaka, H. Segawa and S. Ito, ChemSusChem, 2016, 9, 2604-2608.

12 Y. Rong, X. Hou, Y. Hu, A. Mei, L. Liu, P. Wang and H. Han, Nat. Commun., 2017, 8, 14555.

13 X. Li, M. Tschumi, H. Han, S. S. Babkair, R. A. Alzubaydi, A. A. Ansari, S. S. Habib, M. K. Nazeeruddin, S. M. Zakeeruddin and M. Grätzel, Energy Technol., 2015, 3, 551-555.

14 C.-M. Tsai, G.-W. Wu, S. Narra, H.-M. Chang, N. Mohanta, H.-P. Wu, C.-L. Wang and E. W.-G. Diau, J. Mater. Chem. A, 2017, 5, 739-747.

15 S. G. Hashmi, D. Martineau, M. I. Dar, T. T. T. Myllymaki, T. Sarikka, V. Ulla, S. M. Zakeeruddin and M. Gratzel, J. Mater. Chem. A, 2017, 5, 12060-12067.

16 Z. Xiao, Y. Yuan, Y. Shao, Q. Wang, Q. Dong, C. Bi, P. Sharma, A. Gruverman and J. Huang, Nat. Mater., 2015, 14, 193-198.

17 D.-Y. Son, J.-W. Lee, Y. J. Choi, I.-H. Jang, S. Lee, P. J. Yoo, H. Shin, N. Ahn, M. Choi, D. Kim and N.-G. Park, Nat. Energy, 2016, 1, 16081. 
18 Z. Fan, J. Xiao, K. Sun, L. Chen, Y. Hu, J. Ouyang, K. P. Ong, K. Zeng and J. Wang, J. Phys. Chem. Lett., 2015, 6, 1155-1161.

19 H.-S. Kim, S. K. Kim, B. J. Kim, K.-S. Shin, M. K. Gupta, H. S. Jung, S.-W. Kim and N.-G. Park, J. Phys. Chem. Lett., 2015, 6, 1729-1735.

20 H.-S. Kim and N.-G. Park, J. Phys. Chem. Lett., 2014, 5, 29272934.

21 B. Chen, M. Yang, X. Zheng, C. Wu, W. Li, Y. Yan, J. Bisquert, G. Garcia-Belmonte, K. Zhu and S. Priya, J. Phys. Chem. Lett., 2015, 6, 4693-4700.
22 Y. Shao, Z. Xiao, C. Bi, Y. Yuan and J. Huang, Nat. Commun., 2014, 5, 5784.

23 J. Xu, A. Buin, A. H. Ip, W. Li, O. Voznyy, R. Comin, M. Yuan, S. Jeon, Z. Ning, J. J. McDowell, P. Kanjanaboos, J.-P. Sun, X. Lan, L. N. Quan, D. H. Kim, I. G. Hill, P. Maksymovych and E. H. Sargent, Nat. Commun., 2015, 6, 7081.

24 H. Yu, F. Wang, F. Xie, W. Li, J. Chen and N. Zhao, Adv. Funct. Mater., 2014, 24, 7102-7108.

25 Y. Sheng, A. Mei, S. Liu, M. Duan, P. Jiang, C. Tian, Y. Xiong, Y. Rong, H. Han and Y. Hu, J. Mater. Chem. A, 2018, 6, 23602364. 